\title{
Prevalencia y factores de riesgo asociados al suicidio en países latinoamericanos
}

\author{
Romina Mónica Böttcher ${ }^{1}$ y Cristian Javier Garay ${ }^{2}$
}

\begin{abstract}
RESUMEN
El suicidio es el acto de quitarse intencionalmente la propia vida. Se conoce que este es un hecho multicausal que, en los últimos años, se ha convertido en una de las mayores preocupaciones del sistema de salud. Debido a que la población joven-adulta registra la mayor mortalidad, se realizó una revisión de investigaciones académicas y estadísticas proporcionadas por los ministerios de salud, con el objetivo de conocer las variaciones en las tasas de suicidio a partir del año 2000, y los factores de riesgo asociados a las conductas suicidas en Argentina, Brasil, Chile y Uruguay. Se concluye que en estos países la mortalidad se incrementó a lo largo del tiempo, alcanzando sus puntos máximos en períodos de crisis económica, en coexistencia con factores de riesgo personales e interpersonales.
\end{abstract}

Palabras clave: suicidio, prevalencia, Latinoamérica, factores de riesgo.

\section{Prevalence and factors associated to suicide in Latin American countries}

\begin{abstract}
Suicide is the act of intentionally taking one's life. It's known that this is a multicausal event that, in recent years, has become one of the major concerns of the health system. Due to the fact that the young-adult population registers the highest mortality, this article consisted of a review of academic research and statistics provided by ministries of health, with the objective of knowing the variations in suicide rates from the year 2000, and the risk factors associated with suicidal behaviors in Argentina, Brazil, Chile and Uruguay. It is concluded that in these countries' mortality increased over time, reaching its highest points in periods of economic crisis, in coexistence with personal and interpersonal risk factors.
\end{abstract}

Keywords: suicide, prevalence, Latin America, risk factors.

En los últimos años, la conducta suicida se convirtió en una de las preocupaciones más importantes en el sistema de salud. En una investigación realizada por la World Health Organization (2014), se registraron un millón de suicidios, y aproximadamente diez millones de intentos de suicidio, a nivel

\footnotetext{
${ }_{1}^{1}$ Universidad de Buenos Aires, Argentina; rominabottcher@hotmail.com; http://orcid.org/0000-0002-73426147

2 Universidad de Buenos Aires, Argentina; cristiangaray@psi.uba.ar; http://orcid.org/0000-0003-40828876
} 
global. Alrededor de un tercio de las personas con ideación suicida, proceden al intento de suicidio.

A los fines de la presente revisión, es necesario establecer las diferencias entre ideación suicida, conducta suicida y suicidio. La OMS (Organización Mundial de la Salud, 1992) refiere que la ideación suicida consiste en pensamientos pasivos en los que se quiere estar muerto, o pensamientos activos sobre matarse a sí mismo. El concepto de conducta suicida (Roselló \& Berríos, 2004) refiere a una amplia variedad de conductas, como las amenazas e intentos de suicidio, y las conductas autolesivas sin intencion suicida o parasuicidio.

La Clasificación Internacional de Enfermedades, décima edición (CIE-10) (Organización Mundial de la Salud, 1992), define al suicidio como una muerte por lesión autoinfligida, intencional, deliberadamente iniciado por la persona, y como un medio para obtener un fin deseado. Es un hecho multicausal resultado de la interacción de factores genéticos, biológicos, sociales, psicológicos, ambientales y culturales.

El modelo diátesis-estrés (Zuckerman, 1999) explica las conductas suicidas como el resultado de la interacción entre una predisposición o vulnerabilidad, y experiencias vitales que resultan estresantes para el sujeto. Aunque inicialmente el término diátesis describía únicamente una predisposición genética, en la actualidad se sabe que pueden haber vulnerabilidades cognitivas y sociales. En algunos individuos, la diátesis puede consistir en un solo factor, mientras que en otros está constituida por múltiples componentes. Estos factores de vulnerabilidad permanecen latentes en el individuo $\mathrm{y}$, al interactuar con sucesos negativos o estresantes, aumentan la probabilidad de aparición o mantenimiento de un trastorno. La interacción diátesis-estrés (Ingram \& Luxton, 2005) es dinámica. La diátesis puede incrementarse o disminuir en el tiempo, por lo que la cantidad de estrés necesaria para que se desarrolle una patología también deberá aumentar o decrecer. Si la diátesis está ausente, es decir, si no hay una vulnerabilidad, incluso los factores más estresantes no producirán un efecto en el individuo. Por lo tanto, conforma una condición antecedente necesaria para el desarrollo de un problema o trastorno (Kees van Heeringen, 2012).

Estudios previos muestran que la tasa de mortalidad por suicidio, durante el año 2009 en la región latinoamericana, fue de 5.2 por cada 100.000 habitantes, constituyendo un $7.9 \%$ de las causas de muerte en la región. La mayoría de los suicidios fueron cometidos por personas entre 25 y 44 años (Organización Panamericana de la Salud [OPS], 2014).

En un estudio más reciente, los resultados mostraron una tasa estimada de 45.800 suicidios anuales, de los cuales el $75 \%$ se dio en los países de menor desarrollo. Los grupos etarios que más se vieron afectados fueron los adultos mayores (de 70 años en adelante) con un $22.3 \%$, y los jovenes de 20 a 24 años con un $18.2 \%$. La tasa en los adolescentes varió significantemente en cada país, siendo Uruguay el país con la tasa más alta en toda Latinoamérica, y Brasil el menor (Mascayano et al., 2015).

Esta revisión tiene por objetivo recabar los estudios de prevalencia de suicidio en jóvenes adultos de Argentina, Brasil, Chile y Uruguay publicados a partir del año 2000, y detectar los factores de riesgo asociados. 


\section{METODOLOGÍA}

En el presente trabajo se realizó una revisión de investigaciones académicas, para lo cual se efectuaron búsquedas en las bases de datos Redalyc, SciELO, Google Scholar y PubMed. Las palabras clave introducidas para la realización de la búsqueda fueron: "suicidio", "prevalencia", "latinoamérica", "factores de riesgo". Estas palabras fueron introducidas también en inglés. Asimismo, se tuvieron en cuenta estadísticas proporcionadas por los Ministerios de Salud de los paises estudiados (figura $1)$.

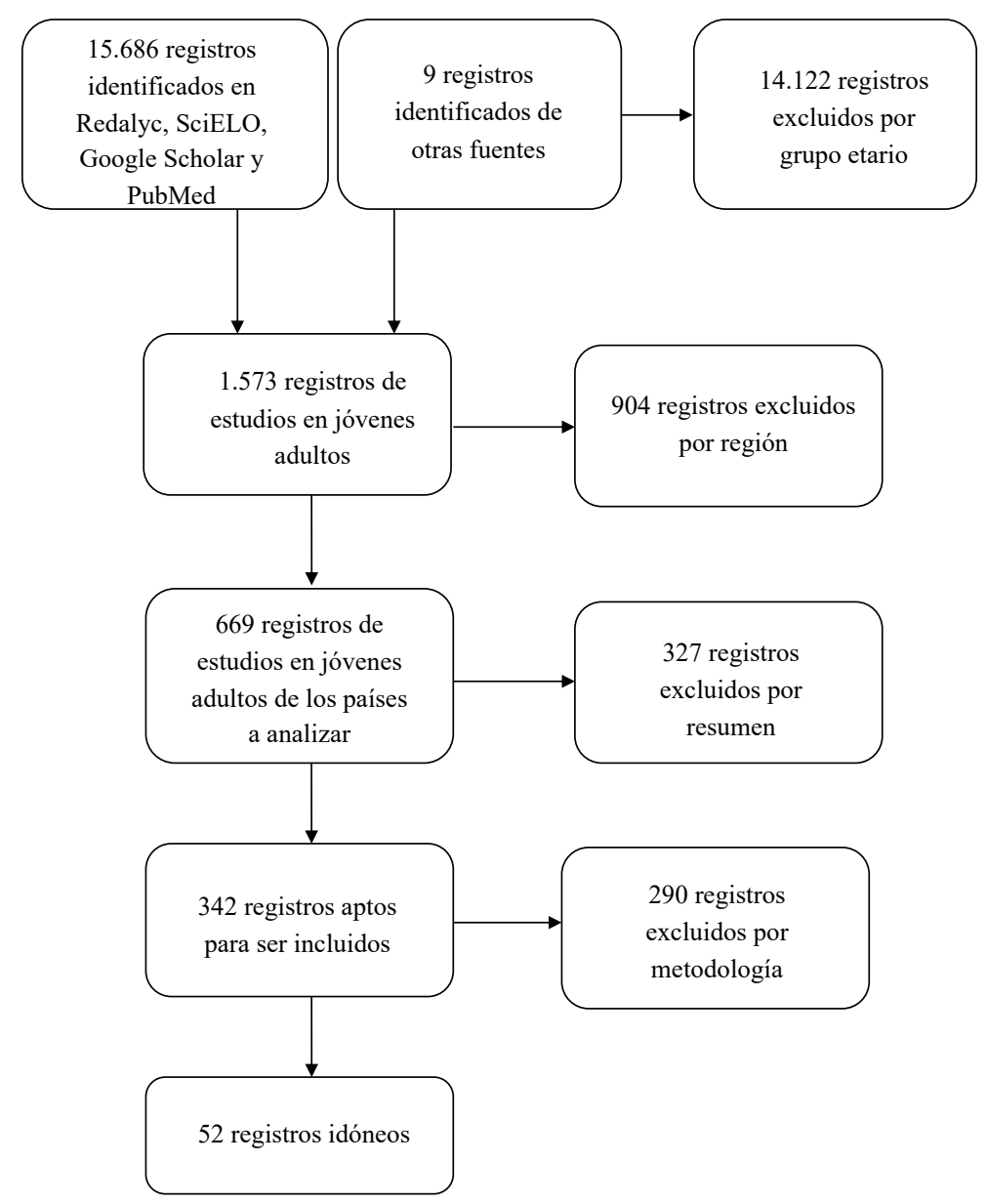

Figura 1. Diagrama de flujo de selección de estudios.

\section{RESULTADOS}

Se han encontrado 8 registros que dan cuenta de la tasa de suicidio en jóvenes adultos en Argentina, 5 registros en Brasil, 3 registros en Chile y 4 en Uruguay entre los años 2000-2015. Por otro lado, 29 estudios resultaron aptos para ser incluidos en el análisis de los posibles factores de riesgo asociados a las conductas suicidas (ver Figura 1).

\section{Prevalencia del suicidio en Argentina}

Altieri (2006) realizó un análisis de las estadísticas proporcionadas por el ministerio de salud, teniendo en cuenta las defunciones registradas en las 
Estadísticas Vitales y proyecciones de población elaboradas por INDEC. En su trabajo dio a conocer que, en el período comprendido entre los años $2000 \mathrm{y}$ 2004, las tasas de mortalidad por suicidio en jóvenes fueron de 10.4 por cada 100.000 habitantes, lo que en relación con años anteriores supuso un aumento en la mortalidad de $75 \%$ en varones y $35 \%$ en mujeres.

En el 2005 (Bella et al., 2012) se registraron 861 muertes por suicidio, en el 2006 hubo un aumento en la mortalidad, alcanzando las 897 defunciones, y en 2007 decreció nuevamente, con 815 suicidios registrados. Entre el 20052007 la tasa media en los jóvenes adultos fue de 8.47 muertes por cada 100.000 habitantes, representando una disminución significante con respecto de las tasas registradas anteriormente. La mayor cantidad de suicidios se dieron en las provincias de Santa Cruz, Salta, Jujuy, Tucumán, Chubut, Neuquén, Catamarca, Formosa, Entre Ríos, Tierra del Fuego y Río Negro. En tanto que los valores más bajos se registraron en Santiago del Estero, Buenos Aires, Misiones, San Luis, San Juan, La Pampa, Santa Fe, Córdoba, Corrientes, Mendoza y La Rioja.

El Ministerio de Salud de la Nación (2010) publicó un boletín especial sobre el suicidio, donde se registró un importante aumento en la mortalidad de jóvenes de 15 a 24 años. En este informe reportaron que en el 2008 la tasa se incrementó a 12.2 muertes por cada 100.000 habitantes. Nuevamente fueron los hombres quienes registraron mayor cantidad de suicidios, con una tasa de 18.5; en tanto que las mujeres representaron una tasa de 6 por cada 100.000 .

Otro boletín epidemiológico publicado por el Ministerio de Salud de la Nación (2013) difundió que en el grupo etario de 15-24 años se registró una tasa de 11.7 muertes por cada 100.000 habitantes en el año 2009, en tanto que en el 2010 volvió a aumentar la cifra alcanzando una tasa de 12.6. En el año 2011 se registró un nuevo incremento en la mortalidad por suicidio en jóvenes, con una tasa de 13 por cada 100.000 habitantes.

La Dirección de Estadísticas e Información en Salud [DEIS] (2013) registró en 2012 un aumento en la tasa respecto del año anterior, con un 14.10. En tanto que en 2013 (Dirección de Estadísticas e Información en Salud, 2014) se produjo una disminución, con una tasa de 13.05 por cada 100.000 habitantes.

United Nations Children's Fund [UNICEF] (2017) reporta que en el año 2014 se registraron 976 fallecimientos por suicidio en personas de entre 15 y 24 años, constituyendo un incremento respecto de años anteriores. Esto representa una tasa de 13.8 muertes por cada 100.000 habitantes. En el 2015 se registró un nuevo incremento, alcanzando una tasa de 14.2.

No se encontraron registros que den cuenta de las tasas de mortalidad por suicidio a partir del año 2016. Sin embargo, se conoce que actualmente el suicidio en Argentina es considerado la segunda causa de muerte en adolescentes y jóvenes adultos, siendo superados únicamente por accidentes de tránsito. UNICEF (2017) reporta que ocurren con mayor frecuencia en hombres, específicamente en aquellos de bajo nivel educativo.

Comparando las tasas de suicidio en jóvenes adultos con las tasas de la población en general (Datos Macro, 2019) podemos ver la evolución de las primeras en relación con la media del país. Hasta el año 2010 las tasas de los jóvenes adultos se mantuvieron por debajo de la media. Sin embargo, a partir de 2011 se registró una mayor mortalidad por suicidio y alcanzó su pico 
máximo en el año 2012, momento en que sobrepasa la tasa promedio (ver figura 2).

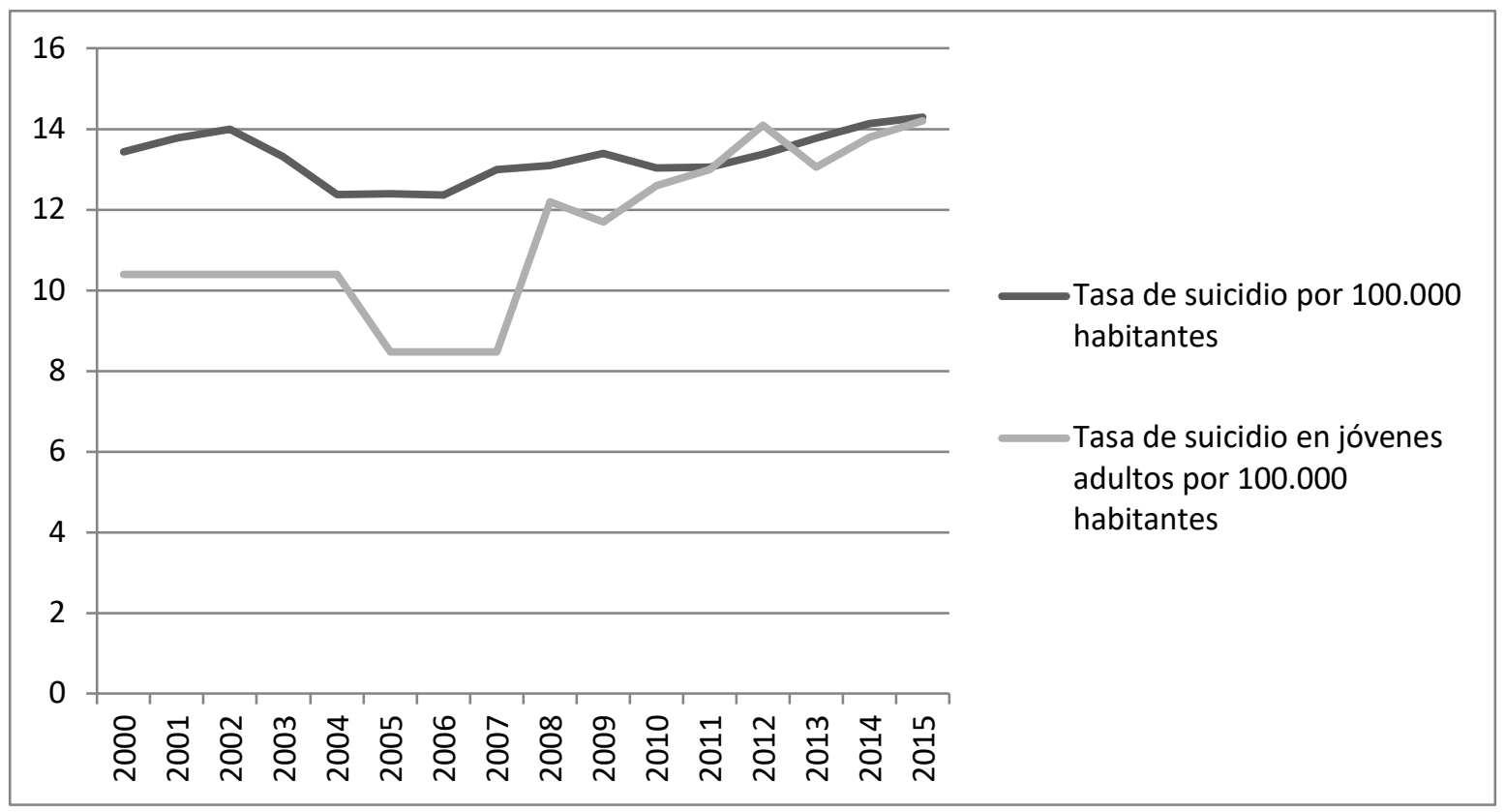

Figura 2. Prevalencia del suicidio en Argentina

\section{Prevalencia del suicidio en Brasil}

Según la Organización Mundial de la Salud, Brasil se encuentra entre los 10 países con más suicidios. Sin embargo, debido a la cantidad de habitantes, las tasas de mortalidad son unas de las más bajas a nivel mundial. La población indígena sería la más afectada, seguida por la población blanca.

Lovisi, Santos Agadir, Legay, Abeja y Valencia (2009) realizaron un estudio de las tasas de mortalidad por suicidio en el país, de acuerdo con los datos provistos por el Sistema de Información de Base de Datos de Mortalidad de Brasil (SIM) y el Departamento del Sistema Único de Salud (DATASUS). En esta investigación reportan que en el periodo 1998-2000 se registró una tasa de 4.4 muertes por cada 100.000 habitantes de 15 a 24 años. Entre los años 2001 y 2003 hubo un incremento en la mortalidad, alcanzando una tasa de 4.8, en tanto que en el período 2004-2006 se registró una tasa de 4.7 por 100.000 habitantes. Los autores indican que el aumento en la mortalidad por suicidio a partir de 2000 en jóvenes adultos se debería, en parte, a la crisis socioeconómica, la pobreza y el desempleo. Asimismo, en otro estudio realizado por Filho y Zerbini (2016), se registró que la población masculina se vería tres veces más afectada que la femenina.

Mendes Ribeiro y Rasga Moreira (2018) realizaron un análisis de las tasas de suicidio según diferentes grupos etarios. En las personas de 15 a 24 años, en el año 2007 se registró una tasa de 4.7 por cada 100.000 habitantes, lo que significaría que se mantuvo constante con relación a los años anteriores. Sin embargo, en el año 2008 se observó un incremento, con una tasa de 5 por 100.000. En los años 2009 y 2010, se produjo un leve descenso en la mortalidad, llegando a una tasa de 4.8 y 4.9 respectivamente. En 2011, por otro lado, se produjo un nuevo aumento alcanzando una tasa de 5 por 100.000 habitantes. 
El ministerio de Salud de Brasil (Ministério da Saúde, 2017) publicó un boletín epidemiológico donde refiere que entre los años 2012 a 2015 hubo un incremento significante en la tasa de suicidio de jóvenes adultos, llegando a un 6.8 por 100.000 habitantes. No se encontraron registros acerca de la mortalidad por suicidio a partir del 2016.

Al realizar la comparación de las tasas por suicidio en jóvenes adultos y la media del país (Datos Macro, 2019), se puede observar una similitud entre ambas hasta el año 2011, en el cual las tasas de mortalidad en los jóvenes se incrementan notablemente con relación a la media, la cual se mantiene relativamente constante (ver figura 3 ).

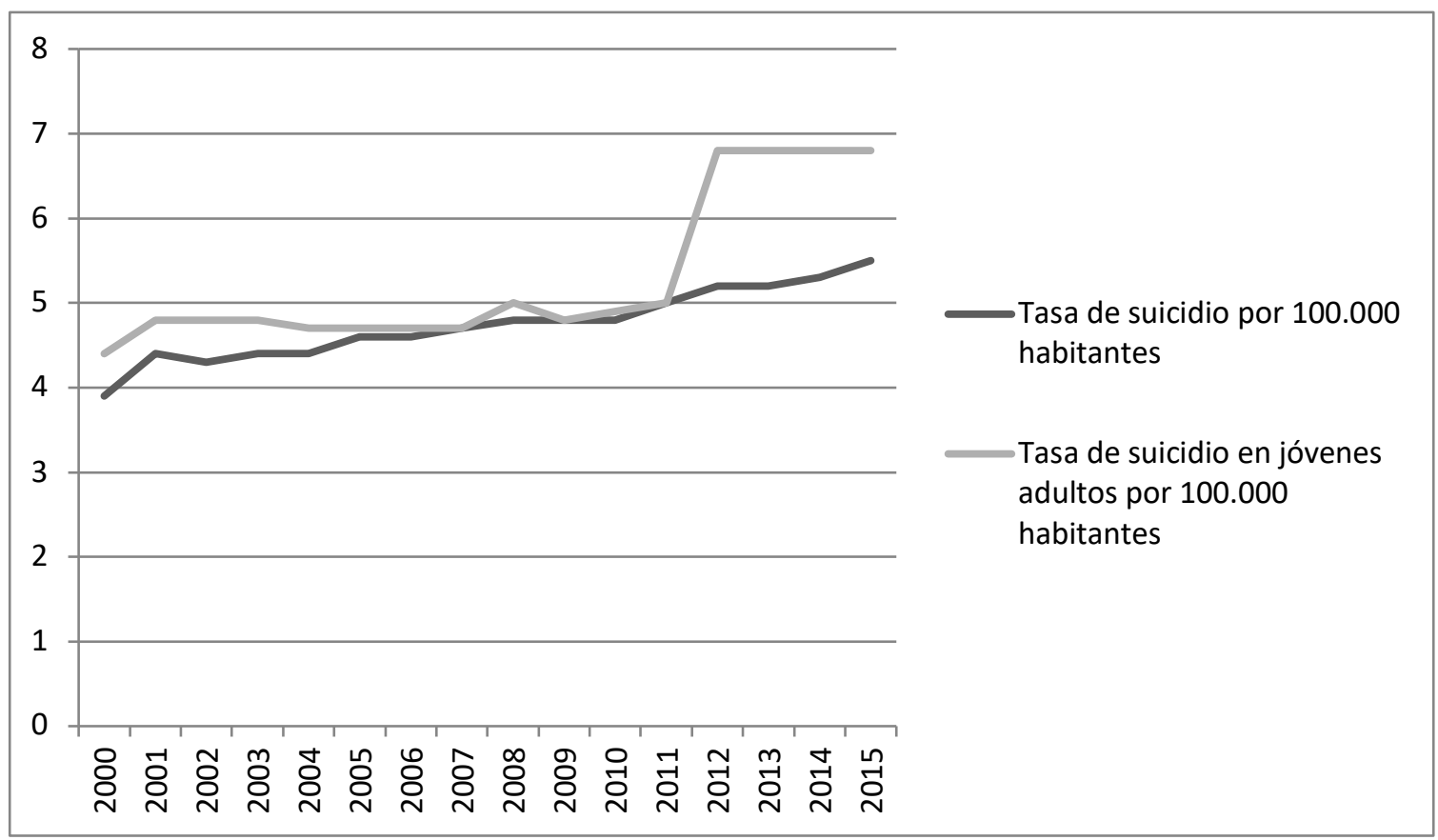

Figura 3. Prevalencia del suicidio en Brasil

\section{Prevalencia del suicidio en Chile}

Otzen, Sanhueza, Manterola y Escamilla-Cejudo (2014) realizaron un estudio de las tasas de mortalidad por suicidio entre los años 1998 y 2009. En su trabajo realizaron una división según grupos etarios, y observaron que el grupo de 15 a 24 años era el que mayor mortalidad registraba. En el año 2000 registraron una tasa de 10.58 por cada 100.000 habitantes. Esta cifra se vio incrementada en 2001, con una tasa de 12.30, en tanto que volvió a decrecer en 2002 y 2003, con tasas de 11.03 y 10.76 por 100.000 habitantes, respectivamente. En el año 2004 se registró un nuevo aumento en la cifra de mortalidad, con una tasa de 12.97. En 2005 se produjo un leve descenso, llegando a una tasa de 11.70 , la cual se mantuvo casi constante en 2006, cuando se registró un 11.63 por 100.000 habitantes. Sin embargo, a partir de 2007 se incrementó la mortalidad por suicidio notablemente, alcanzando una tasa de 14.06. En 2008 un nuevo incremento señaló la mayor tasa de suicidio registrada en los últimos cincuenta años, con una cifra de 15.56 , en tanto que en el 2009 hubo un leve descenso, llegando a 14.69 por cada 100.000 habitantes. 
El Departamento de Estadísticas e Información de la Salud (DEIS, 2017) publicó un informe acerca de la mortalidad por causas externas autoinfligidas en el grupo etario de 15 a 24 años. Aquí reportaron que en el año 2010 se registró una tasa de 14.29 por cada 100.000 habitantes, cifra que fue disminuyendo en adelante. En 2011 la tasa fue de 13.4; en tanto que en 2012 fue 10.79. Esta cifra se mantuvo relativamente constante en los años siguientes, registrándose en 2013 una tasa de 10.15; 10.67 en 2014; y en 2015 un 10.49.

Al igual que en Argentina y Brasil, no fue posible encontrar registros a partir del año 2016. Sin embargo, se estima que en la actualidad el suicidio es la segunda causa de mortalidad en jóvenes. Con relación al género, el Ministerio de Salud reveló que la cifra en hombres es cuatro veces mayor que en las mujeres.

Al realizar la comparación entre la tasa de suicidio en jóvenes y la tasa media del país (Datos Macro, 2019), se puede observar que la primera se encuentra más elevada a lo largo de todo el período analizado. Sin embargo, luego de alcanzar su pico máximo en el año 2008, decreció paulatinamente hasta alcanzar una congruencia con la tasa media de suicidios del país (ver figura 4).

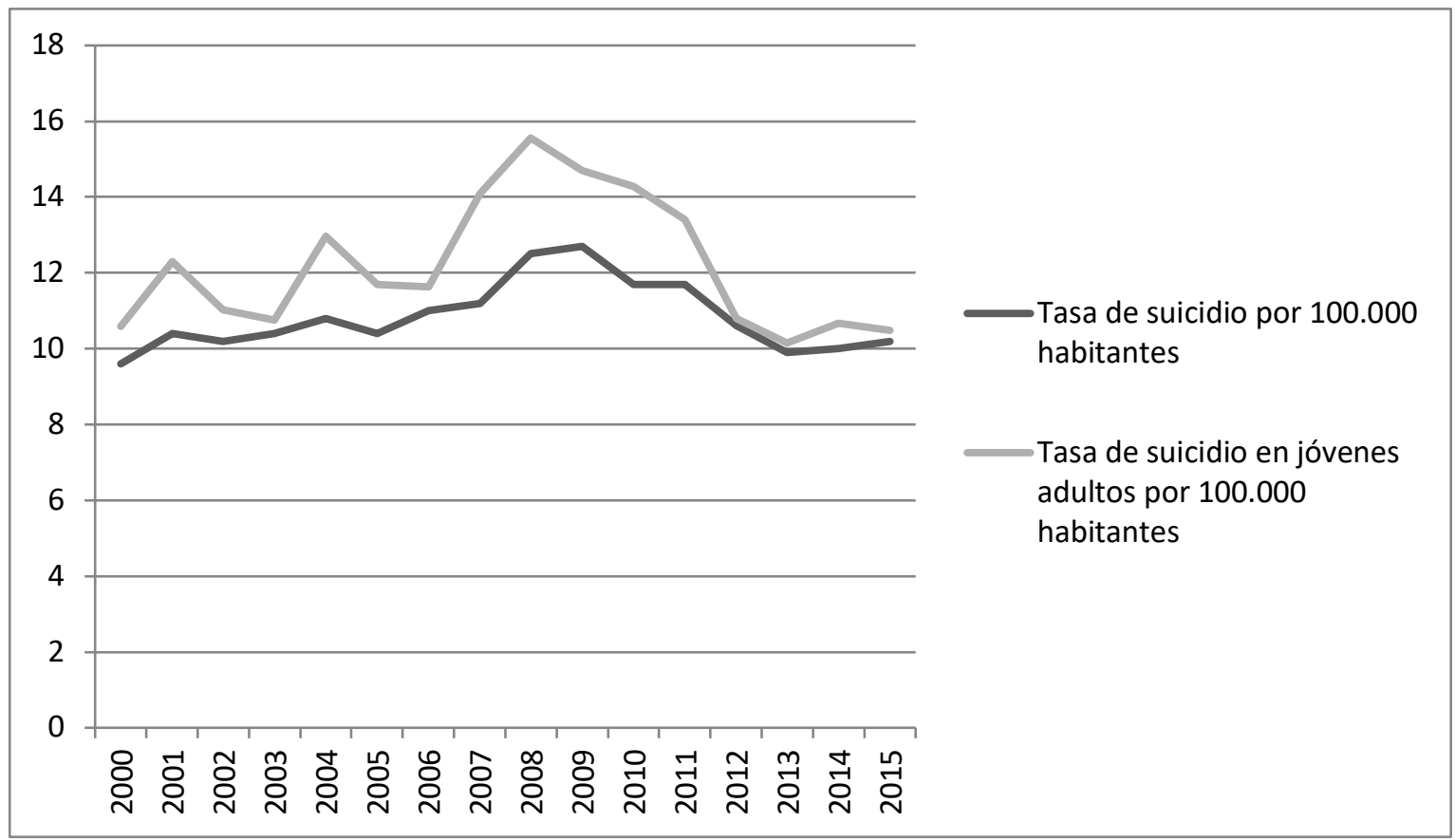

Figura 4. Prevalencia del suicidio en Chile

\section{Prevalencia del suicidio en Uruguay}

La tasa de suicidio en Uruguay es una de las más altas en Latinoamérica. La población más afectada en este país son los adultos mayores de 70 años, seguida por los jóvenes adultos. En estos últimos, el Ministerio de Salud Pública de Uruguay (2013) informó que el suicidio es la causa principal de muerte, y que la cifra registrada en hombres es tres veces mayor que en las mujeres. 
Larrobla et al. (2017) realizaron un estudio evolutivo de las tasas de suicidio en el país, con énfasis en las tasas por grupo etario. En los jóvenes de 15 a 24 años, la mortalidad se mantuvo estable entre los años 1990 y 2002 con una tasa de 13.2 por cada 100.000 habitantes. Sin embargo, a raíz de la crisis económica, entre 2003 y 2008 se registró un incremento en la tasa, alcanzando un 13.9 por 100.000 habitantes. En los años 2009-2014 se produce un importante crecimiento en la mortalidad por suicidio en jóvenes, dando como resultado una tasa de 16.8 por cada 100.000 habitantes.

El Ministerio de Salud (2017) publicó un informe sobre las defunciones por causa externa autoinfligida, donde se reportó que en el año 2015 la tasa fue de 17.51 por 100.000 habitantes, constituyendo la mayor tasa por suicidio registrada hasta entonces. Al igual que en los demás países analizados en este trabajo, no fue posible encontrar registros de las tasas a partir del año 2016.

Comparando la tasa de suicidio en jóvenes adultos con la tasa media de Uruguay (Datos Macro, 2019), se observa que la primera se mantuvo relativamente constante y por debajo de la media hasta el año 2009, cuando comienza a incrementar notablemente, adquieriendo valores similares a la media registrada (ver figura 5).

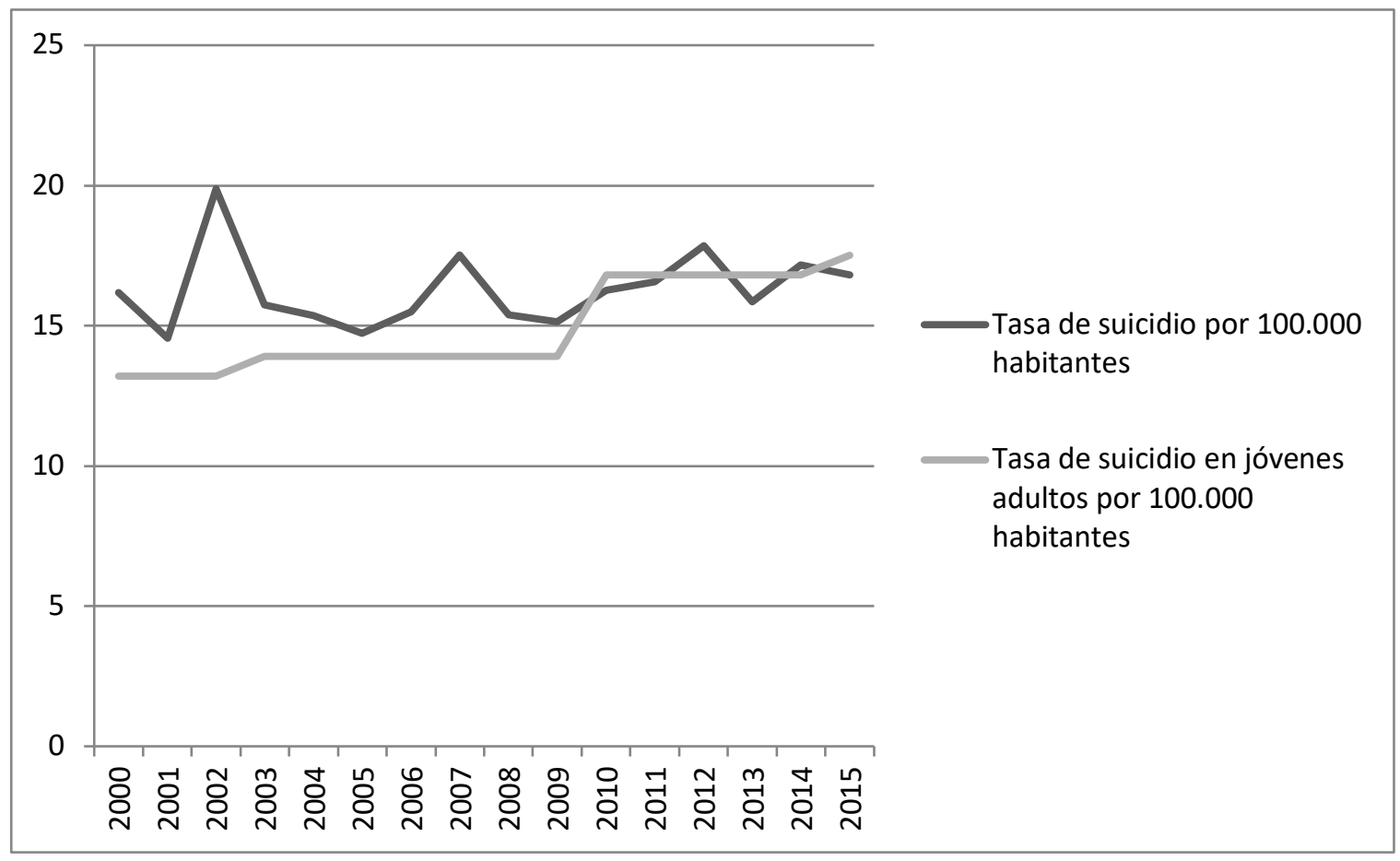

Figura 5. Prevalencia del suicidio en Uruguay

Al comparar los gráficos de los países analizados (ver figura 6 y tabla 1), se puede observar que Brasil es el país que a lo largo del período estudiado mantuvo la menor tasa de suicidio en jóvenes adultos, en tanto que Uruguay fue el país con mayores tasas. En Argentina, Brasil y Uruguay la mortalidad por suicidio se vio incrementada a lo largo del tiempo. Sin embargo, en Chile luego del pico alcanzado en el 2008 las tasas decrecieron notablemente. En ninguno de los países estudiados se encontraron registros que den cuenta de la prevalencia del suicidio en los años posteriores al 2015. 


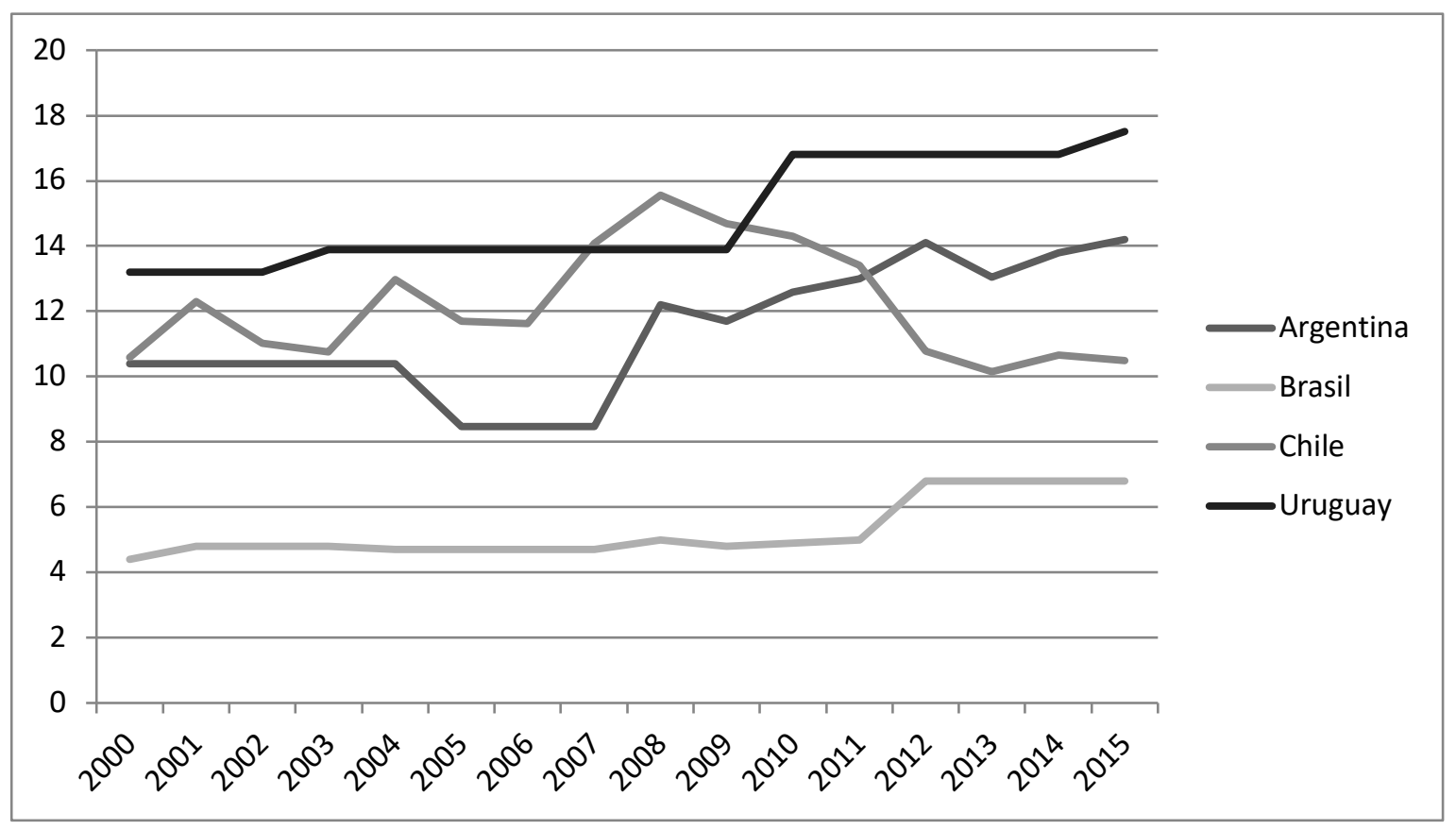

Figura 6. Gráfico comparativo de los datos presentados en Tabla 1.

Tabla 1.

Comparación de prevalencia del suicidio en jóvenes adultos por 100.000 habitantes.

\begin{tabular}{ccccc}
\hline & Argentina & Brasil & Chile & Uruguay \\
\cline { 2 - 5 } 2000 & 10.4 & 4.4 & 10.58 & 13.2 \\
2001 & 10.4 & 4.8 & 12.3 & 13.2 \\
2002 & 10.4 & 4.8 & 11.03 & 13.2 \\
2003 & 10.4 & 4.8 & 10.76 & 13.9 \\
2004 & 10.4 & 4.7 & 12.97 & 13.9 \\
2005 & 8.47 & 4.7 & 11.7 & 13.9 \\
2006 & 8.47 & 4.7 & 11.63 & 13.9 \\
2007 & 8.47 & 4.7 & 14.06 & 13.9 \\
2008 & 12.2 & 5 & 15.56 & 13.9 \\
2009 & 11.7 & 4.8 & 14.69 & 16.8 \\
2010 & 12.6 & 4.9 & 14.29 & 16.8 \\
2011 & 13 & 5 & 13.4 & 16.8 \\
2012 & 14.1 & 6.8 & 10.79 & 16.8 \\
2013 & 13.05 & 6.8 & 10.15 & 16.8 \\
2014 & 13.18 & 6.8 & 10.67 & 16.8 \\
2015 & 14.2 & 6.8 & 10.49 & 17.51 \\
\hline
\end{tabular}

\section{Factores de riesgo asociados a las conductas suicidas}

Antes de analizar los factores que inciden en las conductas suicidas de los jóvenes adultos, es necesario establecer la diferencia entre factores de 
riesgo y protectores. Los factores de riesgo son "las caracteristicas detectables en un individuo, familia, grupo o comunidad que aumentan la probabilidad de experimentar o sufrir un daño" (Burak, 1999, p. 3). Los factores protectores, por otro lado, son aquellas características que mantienen la salud y pueden contrarrestar los posibles efectos de los factores de riesgo (Burak, 1999).

En este apartado, por lo tanto, se analizarán los posibles factores de riesgo. Debido a la variedad de factores que influyen en las conductas suicidas y en la consumación del suicidio, se decidió dividirlos en tres grandes áreas: factores personales, interpersonales y socioeconómicos.

\section{Factores personales}

a. Trastornos mentales: Steele y Doey (2007) realizaron un análisis sobre la epidemiología del suicidio en jóvenes y los factores de riesgo. Descubrieron que el $90 \%$ de los adolescentes que consuman el suicidio sufren de algún trastorno psiquiátrico asociado en el momento de su muerte. Cuantos mayores trastornos comórbidos sufra una persona, mayor será el riesgo de suicidio. Según estos autores, los trastornos depresivos son los más frecuentes, afectando entre el 49\% y 64\% de los jóvenes. En las mujeres el factor de riesgo más significativo es la presencia de depresión mayor, distimia y trastornos alimenticios. Otros trastornos que pueden contribuir a las conductas suicidas son la esquizofrenia y trastorno bipolar. Este estudio y sus resultados fueron comprobados por Hawton y Heeringen (2009), quienes llegaron a la misma conclusión: el 90\% de las personas que se suicidan sufren de uno o varios trastornos mentales comórbidos.

b. Consumo de alcohol y drogas: El inicio en el consumo de alcohol y drogas suele darse en la adolescencia (Organización Mundial de la Salud, 2011). Varios estudios (Adan, 2012; White et al., 2012) señalan que la impulsividad se asocia al consumo excesivo de estas sustancias. Las conductas impulsivas afectan al proceso de toma de decisiones, provocando que se responda rápida e irreflexivamente, acarreando consecuencias negativas a la persona (Adan, 2012).

Jiménez et al. (2005) hicieron un estudio de caso y control, donde compararon un grupo de jóvenes de 15 a 26 años que intentaron alguna vez suicidarse, con personas de la misma edad que nunca lo intentaron. Los resultados indicaron que la media de consumo de alcohol en los casos fue de 3.5 veces por semana, en tanto que en los controles fue de 1.26. Con relación al uso de drogas, la media de consumo en los casos fue de 0.53 veces a la semana, y en los controles 0.20 . De acuerdo con los resultados obtenidos, los jóvenes que refieren hábitos de consumo de alcohol dos o más veces por semana o consumo de drogas una vez por semana, tendrían mayor riesgo de intento suicida.

c. Desesperanza: La desesperanza es una creencia en la cual la persona considera imposible lograr algo en el presente o futuro, percibe constantes imposibilidades y problemas que no se pueden resolver y que lo llevan a resignarse y abandonar sus sueños y ambiciones (Wenzel, Brown, \& Beck, 2009). Beck, Brown, Berchick, Stewart y Steer (1990) realizaron un estudio a 1958 pacientes ambulatorios, donde encontraron que la desesperanza estaba significativamente relacionada con un posible suicidio. Administraron la escala de desesperanza de Beck y usaron una escala de corte de 9 o superior, con lo que pudieron identificar que 16 de cada 17 pacientes $(94,2 \%)$ finalmente se suicidaron. 
En un estudio (Jaiswal et al., 2016) se determinó que la desesperanza se presenta con mayor intensidad durante la ideación suicida, lo que a su vez la mantiene y aumenta el riesgo de suicidio.

d. Orientación sexual: King et al. (2008) realizaron una revisión bibliográfica partiendo de la hipótesis de que las personas que forman parte del colectivo LGBT+ tienen un mayor riesgo de poseer un trastorno mental, ideación y conductas suicidas. Los resultados demostraron que su hipótesis era correcta: los integrantes de dicho colectivo tendrian un riesgo suicida mayor que las personas heterosexuales, así como también una probabilidad 1,5 veces mayor de sufrir trastornos mentales y dependencia de alcohol y sustancias.

Diversos factores se asocian al suicidio en esta población en particular: homofobia, discriminación, bullying, estereotipos, abuso físico y sexual, entre otros.

e. Baja autoestima y autoconcepto: Branden (1995) define la autoestima como la capacidad de considerarse competente frente a desafios de la vida, y sentirse merecedor de felicidad. La alta autoestima está asociada a una vida activa, un gran control de las circunstancias y una buena capacidad para manejar el estrés. La baja autoestima, por el contrario, puede llevar a la apatía, al aislamiento, a una actitud pasiva y un mal manejo del estrés, lo que puede llegar a constituir un factor de riesgo para la aparición de la ideación o de conductas suicidas (Jiménez, Mondragón, \& González-Forteza, 2007). Pinto y Wishman (1996) sostienen que el autoconcepto y la ideación suicida están muy relacionados, y esta asociación es aún mayor en los adolescentes y jóvenes adultos, ya que están en una etapa de transición y cambios donde deben adaptarse a modificaciones en su imagen fisica y de su realidad social. Fernández Pérez y Merino Madrid (2001) realizaron un estudio sobre una muestra de 161 adolescentes de entre 16 y 19 años, cuyo objetivo fue analizar los indices de ideación suicida y su relación con el autoconcepto y la depresión. Para esto aplicaron la escala de ideación suicida, la escala de autoconcepto, y el inventario de depresión de Beck. Los resultados indicaron que las adolescentes tenían niveles más altos de ideación suicida que los adolescentes; mientras en el grupo de las adolescentes la depresión explicaba hasta un 64\% de varianza de ideación suicida. En el grupo de los adolescentes, la variable que explicaba la mayor proporción era el autoconcepto.

\section{Factores interpersonales}

a. Disfunción familiar: Herrera (1997) sostiene que la familia desempeña una función primordial al ejercer las influencias más tempranas y duraderas en la formación de la personalidad de los individuos. Además, actúa como un agente modulador en su relación con el medio, propiciando una menor o mayor vulnerabilidad para la enfermedad y aprendizaje de conductas protectoras.

Guibert Reyes y Torres Miranda (2001) realizaron un estudio analítico retrospectivo de corte transversal, de tipo caso control, con una duración de 5 meses. La muestra estuvo conformada por 31 individuos que cometieron intentos suicidas en ese período, y 31 controles sin antecedentes de tentativa de suicidio o trastornos psiquiátricos. Se utilizó el test FF-SIL que mide el funcionamiento familiar, $y$ se realizaron entrevistas familiares semiestructuradas con participación del mayor número de miembros posible. 
Observaron que los pacientes con intentos de suicidio tenían familias predominantemente disfuncionales, mientras que en el grupo control las familias resultaron predominantemente funcionales. En relación con la adaptabilidad, los sujetos suicidas valoraron a sus familias como poco adaptables a los cambios (67.7\%), en tanto que los controles manifestaron una buena adaptabilidad familiar (87.1\%). Sobre la categoria de afectividad, los casos suicidas reportaron una mala afectividad en sus familias (41.9\%), a diferencia de los controles, en quienes predominan fuertes vínculos afectivos familiares $(51.6 \%)$. En cuanto a la cohesión, el predominio de baja cohesión corresponde a las familias con intento suicida $(70.9 \%)$, y la alta cohesión a familias controles (77.4\%). En relación con la armonía, en los casos se obtuvo una alta desarmonía entre los miembros de la familia $(87.1 \%)$, en tanto que en los controles estaba presente la armonía en el $80.6 \%$ de las familias. Por último, la comunicación en las familias con casos suicidas resultó ser generalmente mala $(54.8 \%)$, mientras que en las familias controles fue generalmente buena (54.8\%).

b. Abuso sexual: Diversos estudios encontraron que, en casos de abuso sexual a adolescentes, las consecuencias a corto plazo abarcan trastornos disociativos, trastorno de estrés postraumático y depresión (Trickett, Noll, Reiffman, \& Putnam, 2001; Noll, Trickett, Susman, \& Putnam, 2006). Otro estudio encontró que es más probable que los adolescentes abusados sexualmente (comparados con otros adolescentes) sufran trastornos alimenticios, consuman drogas y realicen conductas suicidas y autolesivas (Feiring, Miller-Johnson, \& Cleland, 2007).

Por otro lado, el haber sufrido abuso sexual en la infancia o adolescencia también puede provocar sintomatología en adultos. Las víctimas adultas tienen mayor probabilidad de sufrir depresión, ansiedad, baja autoestima e ideación suicida (Guerricaechevarria \& Echeburúa, 2005). Luster y Small (1997) encontraron que el abuso fisico aumenta en mayor medida el riesgo de ideas y conductas suicidas en víctimas de abuso sexual, especialmente en adolescentes que continúan siendo objeto de abuso. Estos autores concluyeron que los hombres adultos que fueron abusados en su infancia cometen más intentos de suicidio y registran mayor tasa de conductas e ideación suicida que las mujeres víctimas de abuso sexual infantil.

c. Aislamiento y soledad: La soledad es un factor de riesgo asociado tanto a alteraciones físicas como psicológicas, como la depresión, conductas suicidas y abuso de alcohol y drogas (Heinrich \& Gullone, 2006). La soledad es, en cierto grado, una experiencia esperable en los adolescentes, en quienes el deseo de estar solos puede ser beneficioso para el desarrollo de su personalidad. Sin embargo, en algunos casos donde se llega a un aislamiento social, puede ocurrir que los sujetos no adquieran las aptitudes necesarias para las interacciones sociales y para enfrentarse o adaptarse a situaciones cambiantes. Otros factores que se asocian a la soledad son sentimientos de baja autoestima, tristeza y desesperanza (Buchholz \& Catton, 1999), los cuales, como se mencionó anteriormente, juegan un papel muy importante en la ideación y conductas suicidas.

d. Relación entre pares y bullying: La relación entre pares y el ambiente escolar son conceptos claves para el desarrollo psicosocial de los jóvenes:

Constituyen un importante contexto para el desarrollo de competencias sociales en el plano emocional y cognitivo, en donde el grupo de amigos y amigas es un espacio necesario para la construcción de su identidad. Las 
amistades cumplen varias funciones en esta etapa, tales como el desarrollo de habilidades sociales, la ayuda para enfrentar las crisis y los sentimientos comunes, la ayuda en la definición de la autoestima y el estatus por la posición del grupo al que pertenecen. Aunado a lo anterior, los grupos de pares son depositarios de los sentimientos que no se quieren compartir con otros (Veladez, Amezcua, González, Montes, \& Vargas, 2011).

Los adolescentes le dan gran importancia a cómo se es percibido por sus pares, por lo que buscan evitar ser señalado como alguien diferente. En ocasiones se dan relaciones de poder, perdiéndose la simetría que debería existir entre pares, y generándose situaciones de maltrato y humillación. Veladez et al. (2011) realizaron una investigación cuantitativa de corte transversal, en la cual participaron 723 adolescentes de entre 12 y 24 años. Se administraron auto-reportes, escala de dificultades escolares, escala de evaluación de aislamiento social, escala de identificación de sentimientos de soledad y abandono, y escala de evaluación de las ideas y pensamientos suicidas. De los alumnos que se identificaron como víctimas, la mayoría refirió haber sido excluido, no poder participar y ser ignorados. En cuanto al maltrato psicológico, refirieron haber recibido apodos descalificantes $(14.9 \%)$, ridiculización (8\%), amenazas $(3.7 \%)$, que hablen mal de ellos $(10 \%)$, y obligarlos a hacer cosas (3.4\%). Del total de sujetos estudiados, 79 de ellos $(9.96 \%)$ manifestaron haber intentado suicidarse y tener ideación suicida. Este estudio permitiría ver la asociación entre procesos de victimización y el incremento del riesgo suicida.

\section{Factores socioeconómicos}

a. Desempleo: Espino Granado (2014) sostiene que la pérdida de empleo constituye uno de los principales factores de riesgo de aparición de problemas de salud mental tales como la ansiedad, el insomnio, la depresión e ideación y conductas suicidas. El desempleo puede causar estos problemas en personas previamente sanas, especialmente en aquellos que se encuentran desempleados hace más de seis meses. Stuckler, Basu, Suhrcke, Coutts y McKee (2009) afirman que en los períodos de recesión económica se encontró una alta correlación entre tasas de desempleo y de suicidio, especialmente entre varones de 30 a 49 años y jóvenes entre 16 y 24.

Esta alta correlación entre el desempleo y el suicidio en épocas de crisis económica puede comprobarse en el análisis de los países latinoamericanos mencionados anteriormente, donde las mayores tasas de suicido correspondian a aquellos años en los que cada país atravesó una crisis de este tipo.

b. Falta de vivienda: Los jóvenes sin hogar son un grupo con gran riesgo de conductas suicidas. Se identificaron varios factores que influyen en este riesgo, siendo un historial de trastornos mentales, abuso sexual y dependencia de alcohol y sustancias los factores más frecuentes (Yoder, Hoyt, \& Whitbeck, 1998).

Kidd (2006) realizó un estudio en el que participaron 208 personas menores de 24 años en situación de calle hace más de un año. Las entrevistas se realizaron en lugares públicos como parques y avenidas. De estos participantes 122 (59\%) fueron hombres, y 86 (41\%) mujeres. Los resultados indicaron que un $46 \%$ de los participantes tuvo al menos un intento de suicidio, y un $76 \%$ reportó haber hecho más de un intento. Con relación al 
género, el $55 \%$ de las mujeres y el $40 \%$ de los hombres afirmaron haber realizado al menos un intento de suicidio.

c. Migraciones: García-Campayo y Sanz Carrillo (2002) realizaron una revisión bibliográfica acerca de los efectos que produce el proceso de migración en la salud mental. Llegaron a la conclusión de que la migración en sí no genera un incremento en el riesgo de padecer trastornos mentales, sino las experiencias traumáticas sufridas durante el proceso migratorio, más aún si se trata de casos de refugiados. Generalmente el estrés se incrementa en el emigrante durante los primeros dos años y posteriormente va disminuyendo hasta alcanzar los valores normales previos. Sin embargo, debido a factores como el shock y conflicto cultural, diferencias de lenguaje, discrepancias entre las expectativas y la situación real, discriminación y pérdida de su red de apoyo, en muchas ocasiones se da la aparición de psicopatología. Entre las principales afecciones psiquiátricas se encuentran: trastorno de estrés postraumático, esquizofrenia, paranoia, ansiedad, somatización y depresión, lo cual como se mencionó anteriormente pueden ser factores de riesgo para la realización de conductas suicidas.

\section{DISCUSIÓN}

En la actualidad el suicidio es una de las principales causas de muerte a nivel global, afectando principalmente a jóvenes, y convirtiéndose en una de las mayores preocupaciones en el sistema de salud.

Como se observó a lo largo de la presente revisión, las tasas de suicidio en jóvenes fueron evolucionando en los últimos años. En Argentina la mortalidad por suicidio se incrementó notablemente en relación con las tasas registradas en los primeros años de este siglo, al punto de convertirse en la segunda causa de muerte en este grupo etario. En Uruguay el suicidio es la primer causa de muerte en jóvenes adultos, y su tasa es la más elevada del continente latinoamericano. Al igual que en Argentina, la mortalidad se vio incrementada a lo largo del período estudiado. En Brasil, si bien la mortalidad también aumentó en gran medida, esto no se ve reflejado en la tasa debido al progresivo crecimiento de la población. En Chile, por el contrario, la mortalidad por suicidio disminuyó luego de alcanzar su pico máximo en el 2008.

En los países mencionados se encontró una coincidencia con respecto al incremento en las tasas de suicidio: se registraron más cantidad de suicidios consumados en aquellos años en que cada país afrentaba una crisis económica, que acarreó consigo un gran nivel de desempleo. Sin embargo, debido a la multicausalidad del suicidio, la ideación y conductas suicidas, sería un reduccionismo considerar solamente al desempleo y la recesión económica como únicos causantes.

Existen múltiples razones por las que una persona decide quitarse la vida, y como se mencionó anteriormente, no todos los estresores generan las mismas respuestas en los sujetos debido al modo en que se procesa la información, y a la presencia o ausencia de una vulnerabilidad, que al interactuar con dichos estresores puede desatar un trastorno o un tipo de conducta riesgosa.

Entre los factores de riesgo que inciden en las conductas suicidas de los jóvenes adultos se destaca principalmente la presencia de trastornos mentales. Fue demostrado en múltiples estudios que aquellas personas que 
sufren de depresión, distima, trastornos alimenticios, bipolaridad, esquizofrenia, entre otros, poseen un mayor riesgo de realizar dichas conductas, y este riesgo se vería acrecentado en aquellos casos donde habría una comorbilidad de trastornos psiquiátricos. En relación con la presencia de depresión y otros trastornos de humor, la desesperanza juega un papel fundamental. Aquí se ve afectada la tríada cognitiva, es decir, que posee una percepción negativa sobre el mundo, sobre sí mismo y su futuro (Beck, Rush, Shaw, \& Emery, 1979). El sujeto considera imposible la resolución de problemas, y se ve obligado a resignar sus sueños y ambiciones, lo que desembocaría en la ideación suicida, y eventualmente en la ejecución de conductas suicidas. En cuanto a la percepción negativa de sí mismo, una baja autoestima y autoconcepto llevaria a una incapacidad de considerarse competente y merecedor de felicidad.

Asimismo, el consumo excesivo de alcohol y la drogodependencia indicarian un riesgo mayor de ejecutar conductas suicidas debido a la impulsividad en la toma de decisiones. Por otra parte, la orientación sexual de los jóvenes también influiría en la ideación y conductas suicidas, siendo el colectivo LGBT+ más susceptible que los sujetos heterosexuales, debido a factores tales como la discriminación y la homofobia.

En relación con los factores interpersonales que influyen en las conductas suicidas, tiene gran relevancia el lugar de la familia y el grupo de pares. Ambos son de gran importancia para el desarrollo de la personalidad e identidad del sujeto, además de conformar su red de apoyo. Cuando la familia resulta disfuncional, hay mala comunicación, poca afectividad, una baja adaptabilidad a cambios, poca armonía y vínculos débiles entre sus miembros, existe una mayor probabilidad de que los jóvenes realicen conductas suicidas. Por otro lado, los jóvenes adultos le dan una gran importancia a cómo es percibido por otros, por lo que quiere evitar ser señalado como diferente por sus pares. Sin embargo, en ocasiones se dan situaciones de bullying, donde el sujeto es maltratado y humillado, llevándolo a sentirse excluido y aislado, lo que podría conducir a la ideación y riesgo suicida. Otro factor de gran importancia es el abuso sexual y el abuso físico: si bien cada uno de estos es un gran factor de riesgo, al ocurrir maltrato físico mientras se es objeto de abuso sexual, se incrementaría aún más el riesgo de realizar conductas suicidas.

Por otra parte, la falta de vivienda y el proceso migratorio también constituyen factores de riesgo. La falta de vivienda, por un lado, frecuentemente se asocia al consumo excesivo de drogas y alcohol, y a situaciones de abuso sexual y físico, que como se mencionó anteriormente resultan en un incremento en el riesgo de conductas suicidas. El proceso migratorio, por otro lado, no genera un riesgo en sí, sino que este aparecería cuando la situación se vuelve traumática para la persona.

Respecto de las limitaciones del presente trabajo, por una parte se debe mencionar la imposibilidad de acceder a estadísticas sobre la mortalidad por suicidio en jóvenes a partir del año 2016. Por otro lado, es necesario destacar la escasa cantidad de estudios realizados en Latinoamérica.

En esta revisión se hizo un análisis sobre los posibles factores de riesgo que inciden en las conductas suicidas. En futuras líneas de investigación podrian desarrollarse los factores protectores que reducen los riesgos aquí tratados. Por otra parte, sería interesante conocer la prevalencia del suicidio en otros países latinoamericanos. 


\section{REFERENCIAS}

Adan, A. (2012). Impulsividad funcional y disfuncional en jóvenes con consumo intensivo de alcohol (binge drinking). Adicciones, 24(1), 17-22. https://doi.org/10.20882/adicciones. 113

Altieri, D. (2006, septiembre). Mortalidad por suicidios en Argentina. Nivel, tendencia y diferenciales. Trabajo presentado en el II Congreso Internacional de Suicidologia, Corrientes, Argentina.

Beck, A. T., Brown, G., Berchick, R. J., Stewart, B. L., \& Steer, R. A. (1990) Relationship between hopelessness and ultimate suicide: a replication with psychiatric outpatients. American Journal of Psychiatry, 147(2), 190-195. https://doi.org/10.1176/ajp.147.2.190

Beck, A. T., Rush, A. J., Shaw, B. F., \& Emery, G. (1979). Terapia cognitiva de la depresión. Bilbao: Desclée De Brouwer

Bella, E., Acosta, A., Villace, B., Lopez de Neira, M., Enders, J., \& Fernández, R. (2012) Análisis de la mortalidad por suicidio en niños, adolescentes y jóvenes. Argentina, 2005-2007. Archivos Argentinos de Pediatria, 111(1), 16-21. https://doi.org/10.5546/aap.2013.16

Branden, N. (1995). Los seis pilares de la autoestima. Buenos Aires: Paidós.

Buchholz, E. S. \& Catton, R. (1999) Adolescents perceptions of aloneness and loneliness. Adolescence, 34(133), 203-13.

Burak, S. D. (1999). Protección, riesgo y vulnerabilidad. Adolescencia Latinoamericana, 1(4), $222-230$.

Datos Macro (2019). Suicidios 2000-2015. Recuperado https://datosmacro.expansion.com/demografia/mortalidad/causas-muerte/suicidio?anio=2015

Departamento de Estadísticas e Información de la Salud (2017). Defunciones y mortalidad por causas. Gobierno de Chile. Recuperado de https://deis.minsal.cl/

Dirección de Estadísticas e Información en Salud (2013). Estadísticas Vitales Año 2012. Ministerio de Salud y Desarrollo Social. Presidencia de la Nación. Recuperado de http://www.deis.msal.gov.ar/index.php/estadisticas-vitales /

Dirección de Estadísticas e Información en Salud (2014). Estadísticas Vitales Año 2013. Ministerio de Salud y Desarrollo Social. Presidencia de la Nación. Recuperado de http://www.deis.msal.gov.ar/index.php/estadisticas-vitales /

Espino Granado, A. (2014). Crisis económica, políticas, desempleo y salud (mental). Revista de la Asociación Española de Neuropsiquiatria, 34(122), 385-404. https://doi.org/10.4321/S021157352014000200010

Feiring, C., Miller-Johnson, S., \& Cleland, C. M. (2007). Potential pathways from stigmatization and internalizing symptoms to delinquency in sexually abused youth. Child Maltreatment, 12, $220-232$. https:/ / doi.org/10.1177/1077559507301840

Fernández Pérez, N. \& Merino Madrid, H. (2001). Predictores de la ideación suicida : un estudio empírico en adolescentes. Revista de Psicopatología y Psicología Clínica, 6(2), 121127. https://doi.org/10.5944/rppc.vol.6.num.2.2001.3909

Filho, M. \& Zerbini, T. (2016). Epidemiologia do suicídio no Brasil entre os anos de 2000 e 2010. Saúde, Ética \& Justiça, 21(2), 45-51.

García-Campayo, J. \& Sanz Carrillo, C. (2002) Salud mental en inmigrantes: el nuevo desafio. Medicina Clinica, 118(5), 187-191. https://doi.org/10.1016/S0025-7753(02)72328-2

Guerricaechevarria, C. \& Echeburúa, E. (2005). Abuso sexual en la infancia: victimas y agresores: un enfoque clinico. Barcelona: Ariel.

Guibert Reyes, W. \& Torres Miranda, N. (2001). Intento suicida y funcionamiento familiar. Revista Cubana de Medicina General Integral, 17(5), 452-460.

Hawton, K. \& van Heeringen, K. (2009) Suicide. Lancet; 373, 1372-1381. https://doi.org/10.1016/S01406736(09)60372-X

Heinrich, L. M. \& Gullone, E. (2006) The clinical significance of loneliness: a literature review. Clinical Psychology Review, 26(6), 695-718. https://doi.org/10.1016/j.cpr.2006.04.002

Herrera, P. M. (1997). La familia funcional y disfuncional, un indicador de salud. Revista Cubana de Medicina General Integral, 13(6), 591-595.

Ingram, R. E. \& Luxton, D. D. (2005) Vulnerability-stress models. Thousand Oaks CA: Sage Publications; Development of Psychopathology: A Vulnerability-Stress Perspective.

Jaiswal, S. V., Faye, A. D., Gore, S. P., Shah, H. R., \& Kamath, R. M. (2016). Stressful life events, hopelessness, and suicidal intent in patients admitted with attempted suicide in a tertiary care general hospital. Journal of Postgraduate Medicine, 62(2),102-104. https://doi.org/10.4103/00223859.180556

Jiménez, A., Mondragón, L., \& González-Forteza, C. (2007) Selfesteem, depressive symptoms, and suicidal ideation in adolescents: Results of three studies. Salud Mental, 30(5), 20-26.

Jiménez, S., Lozano, N, Rodríguez, L., Vargas, A., Rubio,G., \& López, I. (2005) Consumo de alcohol y drogas como factor de riesgo de intento suicida. Medicina Interna Mexico, 21(3), 183-187.

Kees van Heeringen (2012) Stress-Diathesis Model of Suicidal Behavior. En Y. Dwivedi (Ed.), The Neurobiological Basis of Suicide (pp. 113-124). Florida: CRC Press/Taylor \& Francis. https: / / doi.org/10.1201/b12215-7

Kidd, S. A. (2006). Factors Precipitating Suicidality among Homeless Youth: A Quantitative Follow-Up. Youth \& Society, 37(4), 393-422. https://doi.org/10.1177/0044118X05282763

King, M., Semlyen, J., Tai, S., Killaspy, H., Osborn, D., Popelyuk, D., \& Irwin, N. (2008). A systematic review of mental disorder, suicide and deliberate self harm in lesbian, gay and bisexual people. BMC Psychiatry, 8, 1-17. https://doi.org/10.1186/1471-244X-8-70

Larrobla, C., Hein, P., Novoa, G., Canetti, A., Heuguerot, C., Gonzalez, V., Torterolo, M., \& Rodríguez, L. (2017). 70 años de Suicidio en Uruguay: 7 disciplinas, 7 entrevistas, 7 encuentros. Montevideo, 
Uruguay: Ediciones de la Universidad.

Lovisi, G., Santos Agadir, S., Legay, L., Abeja, L., \& Valencia, E. (2009). Análisis epidemiológico del suicidio en Brasil entre 1980 y 2006. Revista Brasileira de Psiquiatria, 31(Suppl II), S86-S94. https://doi.org/10.1590/S1516-44462009000600007

Luster, T. \& Small, S. A. (1997). Sexual abuse history and problems in adolescence: exploring the effects of moderating variables. Journal of Marriage and the Family, 59, 131-142. https://doi.org/10.2307/353667

Mascayano, F., Irrarázabal, M., D’Emilia, W., Shah, B., Varner, S., Sapag, J., Alvarado, R., \& Hsin Yang, L. (2015) Suicide in Latin America: a growing public health issue. Revista de la Facultad de Ciencias Médicas, 73(3), 295-303.

Mendes Ribeiro, J. M., \& Rasga Moreira, M. (2018) Uma abordagem sobre o suicídio de adolescentes e jovens no Brasil. Ciencia \& saude coletiva, 23(9), 2821-2834. https://doi.org/10.1590/141381232018239.17192018

Ministério da Saúde (2017). Boletim Epidemiológico. Suicídio: Saber, agir e prevenir, 48(30), 1-14

Ministerio de Salud (2017). Estadísticas Vitales. Gobierno de Uruguay. Recuperado de https://uins.msp.gub.uy/\#def

Ministerio de Salud de la Nación (2010). Perfil epidemiológico del suicidio en la Argentina, 1988-2008. Boletín de vigilancia de enfermedades no trasmisibles y Factores de Riesgo Nro. 2. Dirección de Promoción de la salud y Control de Enfermedades no Trasmisibles. Presidencia de la Nación.

Ministerio de Salud de la Nación (2013) Estadísticas Vitales. Sistema de Vigilancia Epidemiológica, 5(57). Dirección de Estadísticas e Información en Salud.

Ministerio de Salud Pública de Uruguay (2013). Informe sobre suicidios en adolescentes. Recuperado de https://www.gub.uy/ministerio-salud-publica/sites/ministerio-salud-

publica/files/documentos/publicaciones/Estudio\%20Suicidio\%20en\%20adolescentes\%20y_j\%C3\%B3 venes\%202009.pdf

Noll, J. G., Trickett, P. K., Susman, E. J., \& Putnam, F. W. (2006). Sleep disturbances and childhood sexual abuse. Journal of Pediatric Psychology, 31, 469-480. https://doi.org/10.1093/jpepsy/jsj040

Organización Mundial de la Salud (1992). Clasificación Estadística Internacional de Enfermedades y Problemas Relacionados con la Salud (10 ${ }^{a}$ revisión). Ginebra: Organización Mundial de la Salud.

Organización Panamericana de la Salud (2014). Mortalidad por suicidio en las Américas. Washington, DC: Organización Panamericana de la Salud.

Otzen, T., Sanhueza, A., Manterola, C., \& Escamilla-Cejudo, J.. (2014). Mortalidad por suicidio en Chile: tendencias en los años 1998-2011. Revista médica de Chile, 142(3), 305-313. https://doi.org/10.4067/S0034-98872014000300004

Pinto, A. \& Whisman, M. A. (1996). Negative affect and cognitive biases in suicidal and nonsuicidal hospitalized adolescents. Journal of the American Academy of Child and Adolescent Psychiatry, 35(2),158-165. https://doi.org/10.1097/00004583-199602000-00008

Roselló, J. \& Berríos, M. (2004). Ideación suicida, depresión, actitudes disfuncionales, eventos de vida estresantes y autoestima en una muestra de adolescentes puertorriqueños/as. Interamerican Journal of Psychology, 38(2), 295-302.

Trickett, P. K., Noll, J. G., Reiffman, A., \& Putnam, F. W. (2001). Variants of intrafamilial sexual abuse experience: Implications for long term devel-opment. Journal of Development and Psychopathology, 13, 1001-1019. https://doi.org/10.1017/S0954579401004138

United Nations Children's Fund [UNICEF] (2017). Suicidio. Comunicación, infancia y adolescencia. Guía para periodistas. Red Argentina de Periodismo Científico. Recuperado de https://www.unicef.org/argentina/media/1536/file/Suicidio.pdf

Steele M. M. \& Doey T. (2007) Suicidal behaviour in children and adolescents part I: etiology and risk factors. Canadian Journal of Psychiatry, 52(S1), 21-33.

Stuckler, D., Basu, S., Suhrcke, M., Coutts, A., \& McKee, M. (2009) The public health impact of economic crises and alternative policy responses in Europe: An empirical analysis. Lancet, 374(9686), 315-323. https://doi.org/10.1016/S0140-6736(09)61124-7

Vargas, H. \& Saavedra, J, (2012). Factores Asociados con la conducta suicida en adolescents. Revista de Neuropsiquiatria, 75(1), 19-28. https://doi.org/10.20453/rnp.v75i1.1539

Valadez, I., Amezcua, R., González, N., Montes, R., \& Vargas, V. (2011). Maltrato entre iguales e intento suicida en sujetos adolescentes escolarizados. Revista Latinoamericana de Ciencias Sociales, Niñez y Juventud, 9(2), 783-796.

Wenzel, A., Brown, G., \& Beck, A. T. (2009) Cognitive therapy for suicidal patients. Scientific and clinical applications. Washington, D.C.: American Psychological Association. https://doi.org/10.1037/11862000

White, H. R., Marmorstein, N. R., Crews, F. T., Bates, M. E., Mun, E. Y., \& Loeber, R. (2011). Associations between heavy drinking and changes in impulsive behavior among adolescent boys. Alcoholism: Clinical and Experimental Research, 35(2), 295-303. https://doi.org/10.1111/j.15300277.2010.01345.x

World Health Organization. (2014). Preventing suicide: a global imperative. Recuperado de https://apps.who.int/iris/handle/10665/131056Yoder, K. A., Hoyt, D. R., \& Whitbeck, L. B. (1998). Suicidal behavior among homeless and runaway adolescents. Journal of Youth and Adolescence, 27, 753-771. https://doi.org/10.1023/A:1022813916476

Zuckerman, M. (1999). Diathesis-stress models. Vulnerability to psychopathology: A biosocial model. Washington, DC, US: American Psychological Association. https://doi.org/10.1037/10316-001 
Böttcher, R. M. \& Garay, C. J. / Psicodebate, 21(1), 61 - 78.

Recibido 10-09-2020 | Aceptado 01-12-2020

Este trabajo se encuentra bajo una Licencia Creative Commons Atribución 4.0 Internacional que permite a terceros utilizar lo publicado siempre que se dé el crédito pertinente a los autores y a Psicodebate

Psicodebate, Vol. 21, N 1, Junio 2021 - Noviembre 2021 ISSN: 1515-2251 e-ISSN: 2451-6600 